\title{
Reversible stereodivergent cycloaddition of racemic helicenes to [60]fullerene: a chiral resolution strategy
}

\author{
Rosa M. Girón, ${ }^{\mathrm{a}}$ Jiankun Ouyang, ${ }^{\mathrm{b}}$ Ludovic Favereau, ${ }^{\mathrm{b}}$ Nicolas Vantuyne, ${ }^{\mathrm{c}}$ Jeanne Crassous, ${ }^{\mathrm{b} *}$ Salva- \\ tore Filippone, ${ }^{a *}$ Nazario Martín. ${ }^{\text {a,d* }}$ \\ ${ }^{a}$ Departamento de Química Orgánica, Universidad Complutense de Madrid, Avda. Complutense s/n, E-28040 Madrid, \\ Spain. \\ ${ }^{b}$ Institut des Sciences Chimiques de Rennes UMR 6226, Campus Beaulieu, 35042 Rennes Cedex, France. \\ ${ }^{c}$ Aix Marseille University, CNRS, Centrale Marseille, iSm2, Marseille, France \\ d) IMDEA-Nanociencia, C/ Faraday, 9, Campus de Cantoblanco, E-28049 Madrid, Spain.
}

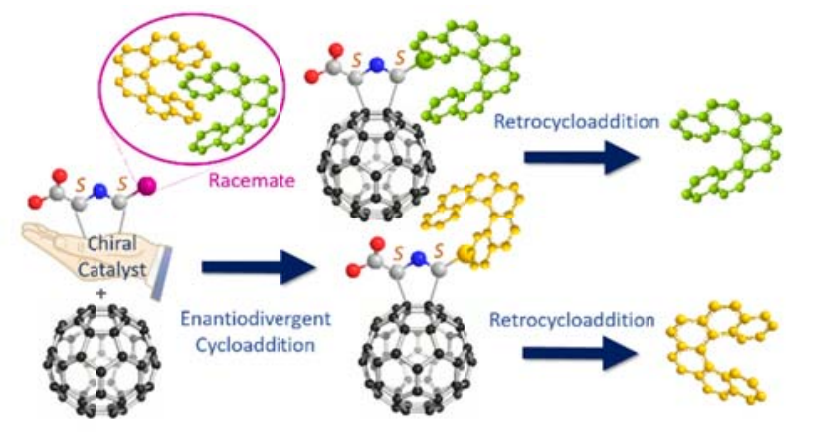

\begin{abstract}
Fullerene and its scarcely-explored reversible covalent chemistry has been harnessed as an efficient alternative for the chiral resolution of racemates. By using only catalytic amounts of chiral agents, stereodivergent 1,3-dipolar cycloadditions of racemic helicenes onto [60]fullerene were carried out. The formed helicene/fullerene diastereomers were easily separated by conventional chromatography and, afterwards, a simple catalyzed 1,3-dipolar retro-cycloaddition afforded helicenes starting materials in high optical purity.
\end{abstract}

Asymmetric (chemical or enzymatic) synthesis, chiral chromatographic separation, kinetic resolution or crystallization methods, are the main methodologies to access to optically active compounds. Far from being out of interest, each one of these strategies are still developing new alternative tools in order to face new challenging chemical problems as well as the constantly high demand of enantiopure chiral compounds.

In this regard, the use of divergent reactions on racemic mixtures (RRM) constitutes a suitable alternative to afford enantiopure compounds to the large number of processes in which both kinetic resolution and asymmetric synthesis failed. ${ }^{1}$ In this strategy, both enantiomers of a racemate chemically interact with a chiral substrate (reagent, catalyst or solvent) giving rise to non-enantiomeric products that could eventually be diastereoisomers (stereodivergent), constitutional isomers (regiodivergent) or different chemical compounds (chemodivergent). Although, neither different reaction rates for both enantiomers are necessary, as in the kinetic resolution, nor stoichiometric amount of chiral reagents, efficient divergent RRM require high selectivity and easily separable products.

On the other hand, chirality has experienced an increasing interest in new fields such as, for instance, nanoscience. ${ }^{2}$ In particular, carbon-based nanostructures such as helicenes, ${ }^{3}$ fullerenes, ${ }^{4}$ or curved polyaromatic hydrocarbons, ${ }^{5}$ feature new optoelectronic properties such as charge-carrier mobility or chiroptical properties, stemming from their chiral arrangement. Actually, for these types of compounds, often lacking functional groups, the enantiomeric chromatographic separation is the only available pathway to obtain optically active compounds. ${ }^{6}$

In the search for alternative racemate resolutions avoiding expensive and time consuming chiral chromatographic separations, we focused our attention on the [60]fullerene covalent chemistry and, in particular, on its less-exploited reversible character. Actually, stereoselective cycloadditions on [60]fullerene by using chiral reagents (asymmetric induction) have been reported. ${ }^{7}$ In this regard, RRM have previously been used, followed by retro-functionalization reactions, to separate chiral fullerenes such as, for instance, $D_{2}-\mathrm{C}_{76}$ and $D_{2}-\mathrm{C}_{84 .}{ }^{8}$ However, to the best of our knowledge, fullerenes have never been previously used for the racemates resolution. Furthermore, from one hand, the introduction of new catalytic methods for the enantioselective functionalization of fullerenes (asymmetric catalysis) allows their application in RRM by using only minor amounts of chiral materials. ${ }^{9}$ On the other hand, the availability of new synthetic tools to carry out retro- 
cycloaddition processes ${ }^{10}$ would enable the recovery of the former racemic starting materials in an enantioenriched form.

Thus, in order to probe the usefulness of this approach, we decided to carry out the enantiodivergent resolution of racemic helicenes, followed by a subsequent retro-cycloaddition reaction, affording the respective isolated enantiomers in an efficient manner, alternative to the use of highly expensive and time consuming chiral HPLC.

Helicenes are non-planar polycyclic aromatic compounds whose helical backbone is formed of ortho-fused aromatic rings. Their extended $\pi$-conjugation combined with their helical topology provide them with high chiroptical properties (huge optical rotations, strong circular dichroic responses and substantial circularly polarized luminescence) and good electronic properties (conductivity and redox properties). ${ }^{3,}{ }^{11}$ For these reasons, they are of great interest for many optoelectronic applications such as chiral organic light-emitting diodes (OLEDs) ${ }^{12}$ transistors, ${ }^{13}$ photovoltaic devices, ${ }^{14}$ chiroptical switches, ${ }^{15}$ or non-linear optical (NLO) materials. ${ }^{16}$ Furthermore, due to their chemical structure based only on aromatic $s p^{2}$ carbon atoms and without functional groups, we considered helicenes a useful racemic benchmark to probe the efficiency of stereodivergent reversible reactions by means of [60]fullerene. In particular, we used 2-formylhexahelicene racemate $\mathbf{1}$ as starting material to carry out the synthesis of the respective helicene-iminoester racemate 2 .

In a first step, racemic 2-hexalicene-iminoesters underwent an enantioselective azomethine ylide 1,3-dipolar cycloaddition reaction to [60]fullerene. Among the available catalytic systems, we chose the pair $\mathrm{Cu}(\mathrm{II})$ acetate $/\left(R_{\mathrm{p}}\right)$-Fesulphos which revealed its efficiency in previous studies directed to the formation of the $(2 S, 5 S)$ pyrrolidino [3,4:1,2][60]fullerene with ee values up to $92 \%$. ${ }^{9}$ Thanks to the high enantiocontrol on the two new formed stereocenters, two diastereomeric helicenepyrrolidino[3,4:1,2][60]fullerenes were formed in good enantiomeric excesses, which were easily separated by conventional silica-gel chromatography. The chiroptical properties of these derivatives, resulting from the curved $\mathrm{Csp}^{2}$ helicene and [60]fullerene moieties, were studied. Finally, the use of a metal-catalyzed retro-cycloaddition reaction under mild conditions $^{10 a, 10 \mathrm{e}}$ afforded both starting 1,3-dipoles which, after insitu hydrolysis, yielded both 2-formylhexahelicene enantiomers which were obtained in good yield and optical purity (Scheme 1).

Scheme 1: Resolution strategy carried out for 2formylhexahelicene racemate 1 .

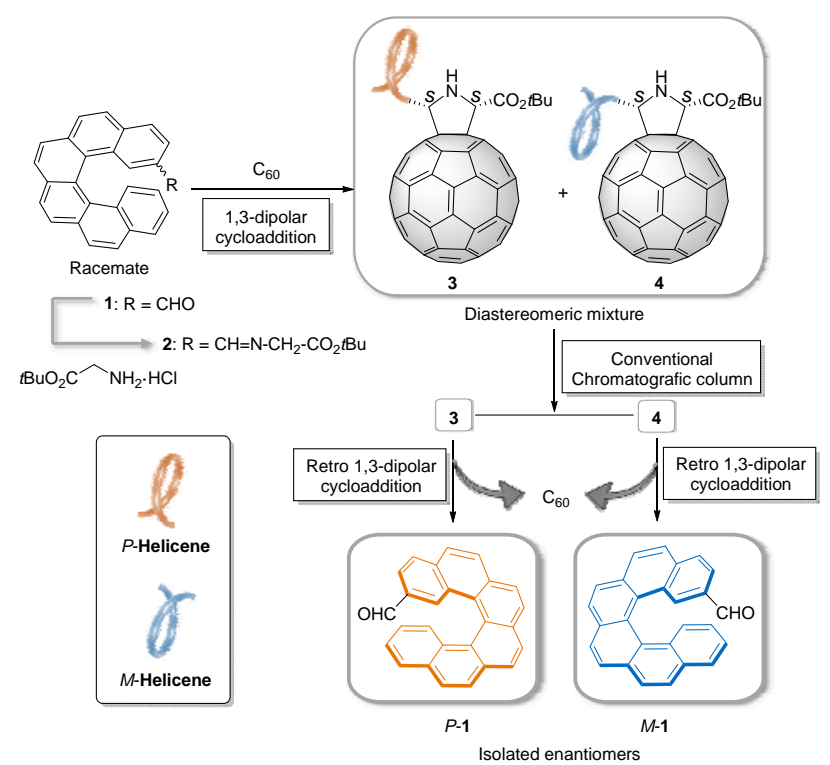

It is important to note that the use of only $10 \%$ of chiral catalyst $\left(\mathrm{Cu}(\mathrm{II})\right.$ acetate/( $\left.R_{p}\right)$-Fesulphos) directed efficiently the cycloaddition of the racemic helicenes 2 onto [60]fullerene affording two diastereoisomers, 3 and $\mathbf{4}$, as result of the high enantiocontrol in the new formed asymmetric carbons of pyrrolidine (the two diastereoisomers differ in the helicenes moiety configuration, while both $\mathrm{C}-2$ and $\mathrm{C}-5$ feature the same configuration, almost completely a $S, S$ configuration, see below). Remarkably, diastereoisomers 3 and $\mathbf{4}$, obtained in a $1: 1$ ratio and in $80 \%$ yield (with respect to helicenes 2) (see Supp. Information, Figure S3), could be easily separated by conventional silica-gel chromatography, thus fulfilling the key requirement for the separation of racemates by stereodivergent RRM.

Each separated diastereoisomer, analysed by chiral HPLC and circular dichroism (CD), showed a very high optical purity: diastereoisomer 4 was obtained in a high enantioenriched form, being ee $>99 \%$ between $(M, S, S)-4$ and $(P, R, R)-4$, (see Supp. Information, Figure S4) while for diastereoisomer 3 the enantiomeric ratio, $(P, S, S)-3$ to $(M, R, R)$-3, was $99 / 1$ (see Supp. Information, Figure S5).

In order to confirm the stereochemical outcome in the racemic series, we carried out the same synthetic study on each of both pure enantiomers $(P$ and $M)$ of helicene $1{ }^{17}$ This study should allow: i) a more easy characterization of the products, and ii) the assessment of the catalytic system to maintain the sign of the asymmetric induction in the presence of another chiral element.

Thus, since 3 is the main product when optically pure $P-2$ underwent the cycloaddition reaction onto [60]fullerene in the presence of $10 \%$ of $\mathrm{Cu}(\mathrm{II})$ acetate $/\left(R_{p}\right)$-Fesulphos, it can be deduced the configuration $(P, S, S)$ for this diastereoisomer. Diastereoisomer 4, formed in a minor amount as result of the unfavorable $(R, R)$ asymmetric induction sense of the catalytic system, features a $(P, R, R)$ configuration. On the contrary and consistently, $(M, S, S)-\mathbf{4}$ is the main product and $(M, R, R)-3$ is the minor one when $M-2$ is used.

Therefore, the catalytic system proved to maintain a high level of stereoselectivity even in the presence of a helicoidal chiral element. It was also observed a slight different behavior with each helicene enantiomers. Indeed, the diastereomeric ratio between $(P, S, S)-3$ and $(P, R, R)-4(92 / 8)$, observed in the 
reaction with iminoester $P$-2, (see Supp. Information, Figure S1) was higher than $(M, S, S)-\mathbf{4} /(M, R, R)-3(83 / 17)$ (see Supp. Information, Figure S2). This is a consequence of the matching of chirality $P$ and chirality of the catalyst $\mathrm{Cu}(\mathrm{II})$ acetate $/\left(R_{\mathrm{p}}\right)-\mathrm{FeSulphos}$ and a mismatching with the chirality $M$ (Scheme 2).

Scheme 2: Cycloaddition reaction of iminoesters $P$-2 and $M-2$ to $\mathrm{C}_{60}$.

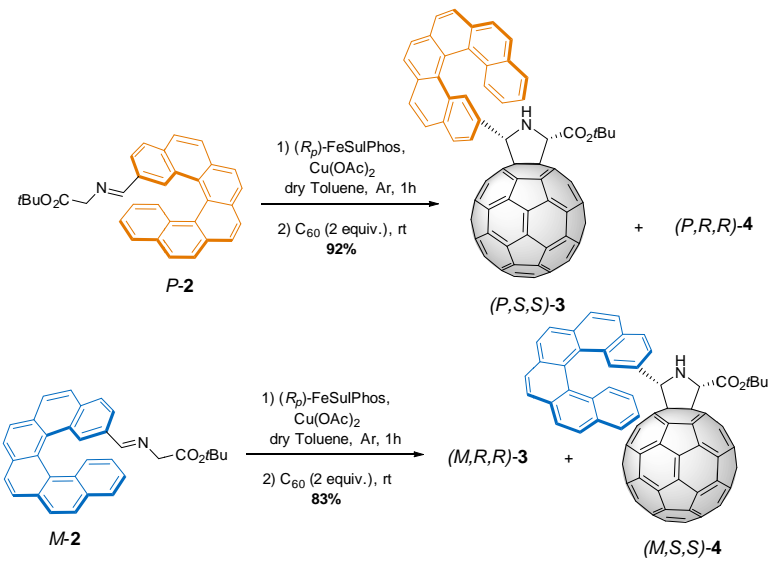

The CD spectra of the four stereoisomers feature both the characteristic peaks of helicene and of [60]fullerene monoadduct and they also confirm the aforementioned asignment. Thus, at $330 \mathrm{~nm}$ the helicene moiety gives rise to an intense peak in the circular dichroism spectrum (Figure 1), with the positive or negative sign corresponding to the enantiomers $P$ or $M$, respectively, while the peak at $427 \mathrm{~nm}$ is the fingerprint of the [60]fullerene monoadducts chirality, which appeared to show a lower intensity (Figure 1, inset), and is used to assign the absolute stereochemistry of each stereoisomer. ${ }^{18}$

Once proved the efficiency of the chiral catalytic system to carry out the stereodivergent RRM, we extended the scope of such functionalization in order to assess the singular reversible covalent chemistry of fullerenes as a useful platform to carry out the racemate resolution. In particular, we have focused our interest on the retro-cycloaddition reaction of pyrrolidino[3,4:1,2][60]fullerenes developed by some of us (retroPrato reaction). ${ }^{10 \mathrm{a}, 10 \mathrm{e}}$ However, despite this reaction gives rise -in a quantitative manner- to pristine [60]fullerene and aldehyde starting materials in refluxing toluene, we had to change the experimental conditions in order to avoid racemization of chiral aldehydes 1 .

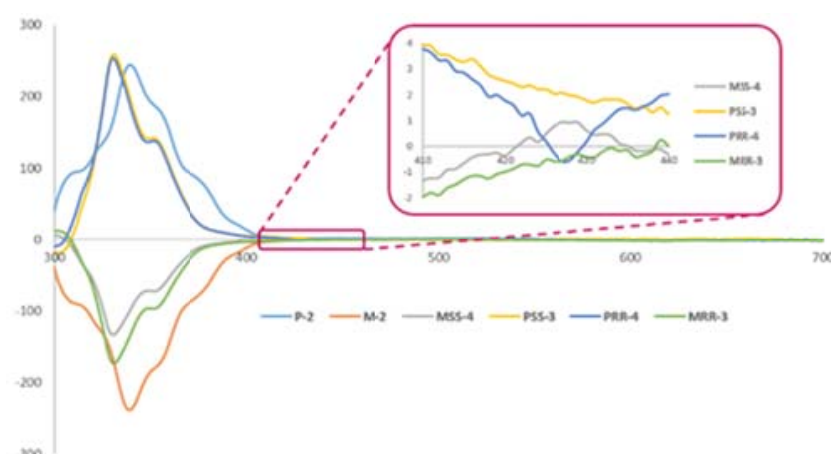

Figure 1. $\mathrm{CD}$ spectra $\left(2 \times 10^{-4} \mathrm{M}\right.$ in $\left.\mathrm{CH}_{2} \mathrm{Cl}_{2}, 25^{\circ} \mathrm{C}\right)$. Inset peak corresponding to [60]fullerene chirality.
We eventually found in the pair $\mathrm{Cu}\left(\mathrm{ClO}_{4}\right)_{2} /$ dppe in refluxing THF $\left(70^{\circ} \mathrm{C}\right)$ the suitable conditions to reverse the cycloaddition reaction in good yields and maintaining the enantiomeric excess. Thus, helicene starting material $P \mathbf{- 1}$ was obtained in a $78 \%$ yield and $e e=99 \%$ from the separated diastereoisomer 3 $[99 \%$ ee, $(P, S, S)-3]$, thus maintaining unchanged the optical purity (see Supp. Information, Figure S8). Analogously, the retro-cycloaddition of 4 [92\% ee, $(M, S, S)]$ affords the helicene $M$ with $82 \%$ yield although the ee was slightly lower, $80 \%$ ee (see Supp. Information, Figure S9) (Scheme 3).

Scheme 3. Enantiospecific retrocycloaddition reaction.

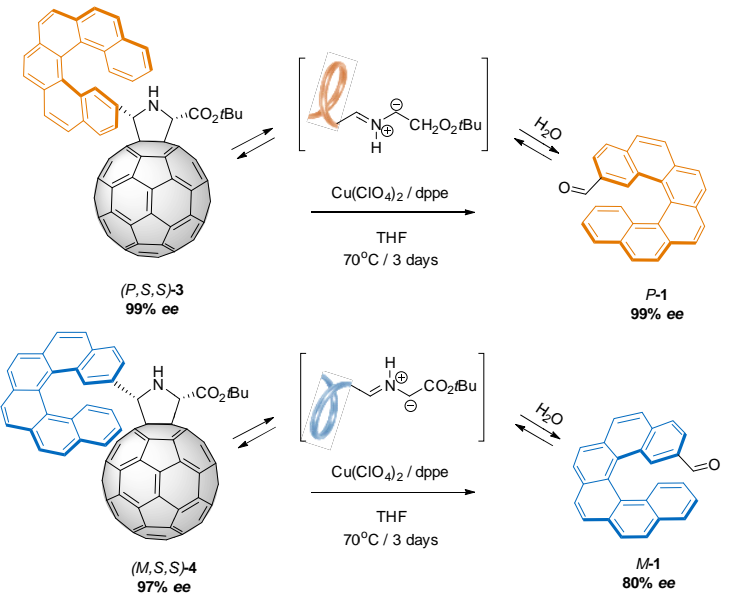

In summary, we report for the first time on the use of the less-explored "reversible" covalent chemistry of [60]fullerene as a useful alternative to carry out the resolution of racemic species. In the representative studied example, the resolution of the racemate of a pyrrolidino[3,4:1,2][60]fullerene endowed with helicenes is described. It involves the reversible stereodivergent 1,3-dipolar cycloaddition reaction of racemic helicenes onto [60]fullerene -employing only catalytic amounts of chiral agents- affording two diastereomeric helicenepyrrolidino[3,4:1,2][60]fullerenes in excellent enantiomeric excesses. Subsequent conventional silica-gel chromatography diastereoisomers separation and metal-catalyzed retrocycloaddition reaction yields the starting 1,3-dipoles whose hydrolyses lead to the starting 2-formylhelicene enantiomers in good yields and excellent optical purity. The above protocol represents a general and efficient method which validates fullerenes for racemates resolution and, in particular, for their efficiency in the resolution of these singular helicene systems.

\section{AUTHOR INFORMATION}

\section{Corresponding Author}

* Email: salvatore.filippone@ucm.es;

* Email: jeanne.crassous@univ-rennes 1.fr

* Email: nazmar@ucm.es

\section{ACKNOWLEDGMENT}

NM acknowledges financial support by the European Research Council (ERC-320441-Chirallcarbon), the Ministerio de Economía y Competitividad (MINECO) of Spain (project CTQ201452045-R) and the Comunidad Autónoma de Madrid 
(PHOTOCARBON project S2013/MIT-2841). R.M.G. thanks the Spanish MECD for a FPU grant (FPU13/03677). JO thanks the Chinese Scholarship Council for financial support. LF and JC thank the CNRS and the University of Rennes 1.

\section{REFERENCES}

(1) (a) Russell, T. A.; Vedejs, E., Enantiodivergent Reactions: Divergent Reactions on a Racemic Mixture and Parallel Kinetic Resolution. In Separation of Enantiomers, Wiley-VCH Verlag GmbH \& Co. KGaA2014; pp 217-266.(b) Miller, L. C.; Sarpong, R. Chem. Soc. Rev. 2011, 40, 4550-4562.

(2) Wang, Y.; Xu, J.; Wang, Y.; Chen, H. Chem. Soc. Rev. 2013, 42, 2930-2962.

(3) (a) Gingras, M. Chem. Soc. Rev. 2013, 42, 1051-1095. (b) Y. Shen, C.-F. Chen, Helicenes Chemistry: From Synthesis to Applications, Springer, Berlin, 2017.

(4) (a) Thilgen, C.; Diederich, F. Chem. Rev. 2006, 106, 5049-5135; (b) Hizume, Y.; Tashiro, K.; Charvet, R.; Yamamoto, Y.; Saeki, A.; Seki, S.; Aida, T. J. Am. Chem. Soc. 2010, 132, 6628-6629.

(5) (a) Rickhaus, M.; Mayor, M.; Juricek, M. Chem. Soc. Rev. 2016, 45, 1542-1556; (b) Rickhaus, M.; Mayor, M.; Juricek, M. Chem. Soc. Rev. 2017, 46, 1643-1660.

(6) Gingras, M.; Felix, G.; Peresutti, R. Chem. Soc. Rev. 2013, 42, 1007-1050.

(7) (a) Novello, F.; Prato, M.; Da Ros, T.; De Amici, M.; Bianco, A.; Toniolo, C.; Maggini, M. Chem. Commun. 1996, 903; (b) Bianco, A.; Maggini, M.; Scorrano, G.; Toniolo, C.; Marconi, G.; Villani, C.; Prato, M. J. Am. Chem. Soc. 1996, 118, 4072; (c) Maggini, M.; Scorrano, G.; Bianco, A.; Toniolo, C.; Prato, M. Tetrahedron Lett. 1995, 36, 2845-2846; (d) Vasella, A.; Uhlmann, P.; Waldraff, C. A. A.; Diederich, F.; Thilgen, C. Angew. Chem. Int. Ed. 1992, 31, 1388-1390 (e) Nierengarten, J.-F.; Gramlich, V.; Cardullo, F.; Diederich, F. Angew. Chem., Int. Ed. Engl. 1996, 35, 2101-2103; (f) Djojo, F.; Hirsch, A. Chem. Eur. J. 1998, 4, 344-356; (g) Illescas, B.; Rifé, J.; Ortuño, R. M.; Martín, N., J. Org. Chem., 2000, 65, 6246-6248; (h) Giacalone, F.; Segura, J. L.; Martín, N., J. Org. Chem., 2002, 67, 3529-3532; (i) Illescas, B. M.; Martín, N.; Poater, J.; Sola, M.; Aguado, G. P.; Ortuño, R. M. J. Org. Chem., 2005, 70, 6929-6932.

(8) (a) Kessinger, R.; Crassous, J.; Herrmann, A.; Rütimann, M.; Echegoyen L. and Diederich F. Angew. Chem. Int. Ed. Engl., 1998, 37, 1919-1922; (b) Crassous, J.; Rivera, J.; Fender, N. S.; Shu, L.; Echegoyen, L.; Thilgen, C.; Herrmann A. and Diederich F. Angew. Chem. Int. Ed. Engl., 1999, 38, 1613-1617.

(9) (a) Filippone, S.; Maroto, E. E.; Martín-Domenech, A.; Suárez, M.; Martín, N. Nat. Chem. 2009, 1, 578; (b) Maroto, E. E.; de Cózar, A.; Filippone, S.; Martín-Domenech, A.; Suárez, M.; Cossío, F. P.; Martín, N. Angew. Chem., Int. Ed. 2011, 50, 6060-6064 ; (c) Maroto,
E. E.; Filippone, S.; Martín-Domenech, A.; Suárez, M.; Martín, N. J. Am. Chem. Soc. 2012, 134, 12936-12938; (d) Maroto, E. E.; Filippone, S.; Suárez, M.; Martínez-Álvarez, R.; de Cózar, A.; Cossío, F. P.; Martín, N., J. Am. Chem. Soc., 2014, 136, 705-712; (e) MarcoMartínez, J.; Reboredo, S.; Izquierdo, M.; Marcos, V.; López, J. L.; Filippone, S.; Martín, N., J. Am. Chem. Soc., 2014, 136, 2897-2904; (g) Maroto, E. E.; Izquierdo, M.; Reboredo, S.; Marco-Martínez, J.; Filippone, S.; Martín, N. Acc. Chem. Res. 2014, 47, 2660-2670.

(10) (a) Martín, N.; Altable, M.; Filippone, S.; Martín-Domenech, A.; Echegoyen, L.; Cardona, C. M. Angew. Chem. Int. Ed. 2006, 45, 110.(b) Martín, N.; Altable, M.; Filippone, S.; Martín-Domenech, A.; Martínez-Álvarez, R.; Suarez, M.; Plonska-Brzezinska, M. E.; Lukoyanova, O.; Echegoyen, L. J. Org. Chem. 2007, 72, 3840-3846. (d) Martín, N.; Altable, M.; Filippone, S.; Martín-Domenech, A Synlett 2007, 2007, 3077-3095. (e) Filippone, S.; Barroso, M. I.; Martín-Domenech, Á.; Osuna, S.; Solà, M.; Martín, N. Chem. Eur. J. 2008, 14, 5198-5206. See also: Lukoyanova, O.; Cardona, C. M. Altable, M.; Filippone, S.; Martín-Domenech, A.; Martín, N.; Echegoyen, L., Angew. Chem. Int. Ed., 2006, 45, 7430-7433.

(11) (a) Saleh, N.; Shen, C.; Crassous, J. Chem. Sci. 2014, 5, 36803694. (b) Isla, H.; Crassous, J. Compt. Rend. Chim. 2016, 19, 39-49.

(12) (a) Yang, Y.; da Costa, R. C.; Smilgies, D.-M.; Campbell, A. J.; Fuchter, M. J. Adv. Mater. 2013, 25, 2624-2628. (b) Brandt, J. R.; Wang, X.; Yang, Y.; Campbell, A. J.; Fuchter, M. J. J. Am. Chem. Soc. 2016, 138, 9743-9746.

(13) Yang, Y.; da Costa, R. C.; Fuchter, M. J.; Campbell, A. J. Nat Photon 2013, 7, 634-638.

(14) Josse, P.; Favereau, L.; Shen, C.; Dabos-Seignon, S.; Blanchard, P.; Cabanetos, C.; Crassous, J. Chem. Eur. J. 2017, 23 , 6277-6281.

(15) (a) Schweinfurth, D.; Zalibera, M.; Kathan, M.; Shen, C.; Mazzolini, M.; Trapp, N.; Crassous, J.; Gescheidt, G.; Diederich, F. J. Am. Chem. Soc. 2014, 136, 13045-13052. (b) Shen, C.; Loas, G. h.; Srebro-Hooper, M.; Vanthuyne, N.; Toupet, L.; Cador, O.; Paul, F.; López Navarrete, J. T.; Ramírez, F. J.; Nieto-Ortega, B.; Casado, J.; Autschbach, J.; Vallet, M.; Crassous, J. Angew. Chem. Int. Ed. 2016, $55,8062-8066$.

(16) Verbiest, T.; Elshocht, S. V.; Kauranen, M.; Hellemans, L.; Snauwaert, J.; Nuckolls, C.; Katz, T. J.; Persoons, A. Science 1998, 282, 913-915.

(17) Moussa, M. E. S.; Srebro, M.; Anger, E.; Vanthuyne, N.; Roussel, C.; Lescop, C.; Autschbach, J.; Crassous, J. Chirality 2013, 25, 455-465.

(18) Marco-Martínez, J.; Marcos, V.; Reboredo, S.; Filippone, S.; Martín, N. Angew. Chem. Int. Ed. 2013, 52, 5115-5119. 